\title{
Identifying and prioritizing the drivers of the future of public hospitals in Iran
}

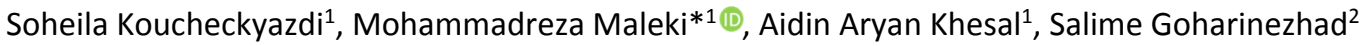

Received: 5 Dec 2019

Published: 12 Sep 2020

\section{Abstract}

Background: Rapid development in today's modern world have made predicting the future difficult. Hospitals, as a major complex in a community, are no exception. Knowing the future facilitates laying plans for it. To predict the future, knowing the factors affecting the future is crucial. This study was therefore conducted to identify and predict the factors affecting the future of hospitals in Iran.

Methods: This study was conducted in three steps. The drivers of the future of hospitals in Iran were listed by analyzing the results of a literature review and then semi-structured interviews with hospital experts. Afterward, the effect of individual drivers was determined using the Fuzzy Analytical Hierarchy Process (FAHP) by formulated in excel software.

Results: Based on the results of the first Phase, 30 effective drivers were identified in 6 areas of modern technology, economy, politics, demography and community, environment, and culture. Prioritization results showed that modern technology (0.246) and economic factors (0.198) had priority over the other factors. Moreover, the effects of sub-factors such as modern information technology and technologies on the future of public hospitals in Iran were more significant compared to those of the other factors.

Conclusion: The present results showed that information technology and modern technology, as well as the economy, were the two main drivers of the future of public hospitals in Iran. Hospitals should identify and lay plans for the mechanisms through which the two factors affect hospitals, given their failure to control the upward trends of the factors.

Keywords: Foresight, Public hospitals, Driving forces, Iran

Conflicts of Interest: None declared

Funding: Iran University of Medical Sciences

\section{*This work has been published under CC BY-NC-SA 1.0 license.}

Copyright $\odot$ Iran University of Medical Sciences

Cite this article as: Koucheckyazdi S, Maleki M, Aryan Khesal A, Goharinezhad S. Identifying and prioritizing the drivers of the future of public hospitals in Iran. Med J Islam Repub Iran. 2020 (12 Sep);34:119. https://doi.org/10.47176/mjiri.34.119

\section{Introduction}

Healthcare systems seek to adapt to momentary developments in today's world of rapid changes, as is the case of other economic and social sectors active in developing countries. Given the allocation of a significant portion of healthcare resources to hospitals, these developments have

Corresponding author: Dr Mohammadreza Maleki, maleki.mr@iums.ac.ir

1. Department of Health Services Management, School of Health Management and Information Sciences, International Campus, Iran University of Medical Sciences, Tehran, Iran

2. Preventive Medicine and Public Health Research Center, Psychosocial Health Research Institute, Iran University of Medical Sciences, Tehran, Iran been assigned to these key components of health service delivery in most countries $(1,2)$. The need for redesigning hospitals and adapting them to anticipated future changes is a widely shared view between different countries (3-5).

Iran is currently facing dual health issues (synchronizing

$\uparrow$ What is "already known" in this topic:

Public hospitals play a key role in any society. Multiple factors affect the future of public hospitals. This study adopts the STEEP framework to scan the key social, technological, environmental, economic, and political issues that may impact the future of public hospitals.

$\rightarrow$ What this article adds:

A convergence of drivers is shaping the future of hospitals. Among them, technology development has a significant role in hospital futures. Progress in health technologies may change the concept of today's hospitals. Scanning and analyzing these factors help the hospitals to embrace a broad range of services with the adaptation of technology. 
contagious with noncontagious diseases) due to increased life expectancy, demographic changes, and epidemiology of health risk factors and the development of modern medical technologies, increasing income and social welfare (6, 7). The failure of the healthcare delivery system in Iran to respond well to this duality suggests a need for radical changes (8).

Evidence suggests little prospect of promoting and enhancing the required financial resources for healthcare and hospitals significantly contribute to healthcare costs in most countries, as confirmed by a WHO report estimating that hospitals in third world countries account for $50-80 \%$ of current government spending on health. In many countries, hospitals budget is, however, never balanced by the production and delivery of their actual services. The health share of global gross domestic product increased from $3 \%$ in the 1950 s to $10 \%$ in the present (9).

Given the importance of technological and social developments, a new approach to the future should be taken and decision makers need to acquire new skills to cope with technological changes (10). Given today's rapid and uncertain environmental changes, foreseeing to deal with possible future changes is becoming increasingly needed (11).

Future research should be performed in an interdisciplinary manner (12). Social science is an interdisciplinary program, as it uses different disciplines to obtain findings and propositions. To be pragmatic, social science is designed to shape the future by seeking to provide human with the information needed for action. Future research is evolving, despite the process of specialization and separation of various disciplines and professions (13). The subject of foresight covers sectors such as culture, economy, politics, health, technology and the arts, and with the upcoming changes, these topics are on the rise in number $(14,15)$.

Identifying the factors affecting the future is the main step in foresight. This study identified the factors affecting the future of hospitals in Iran and determined their effects.

\section{Methods}

\section{Type of research}

This study is part of a prospective study on the foresight for public hospitals in Iran. This part of the study was conducted as a quantitative-qualitative study to identify the factors affecting the future of hospitals in Iran. In the first phase, the results of previous studies and open and structured interviews with hospital experts were identified. Given the unequal effects of the drivers on the future of the hospitals, the second phase determined the effect of individual drivers using the FAHP. These steps are summarized in Figure 1.

\section{Phases 1 and 2: Literature review and interviews}

The main focus of this phase was to identify key driving forces affecting the future of public hospitals through a comprehensive literature review as well as interviews.

\section{Research sample}

At the review stage, the texts of all the documents, reports, articles, theses and books were extracted by topic

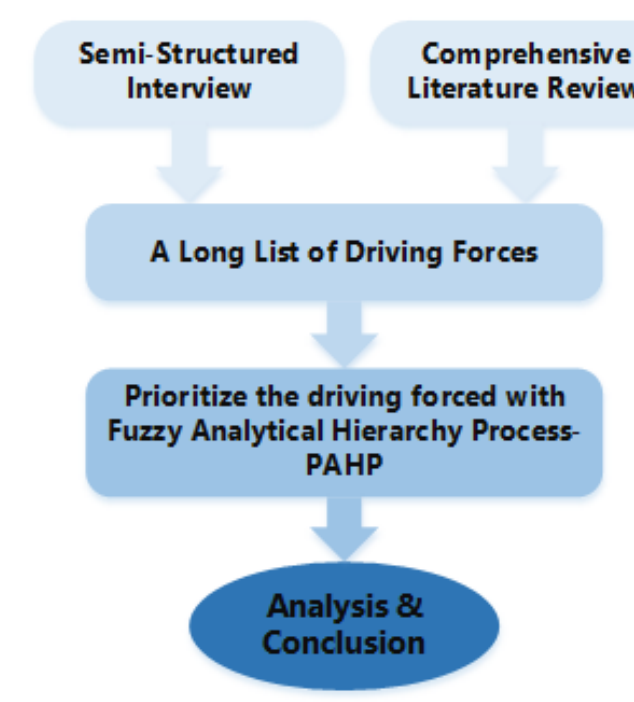

Fig. 1. Research steps

from scientific databases, including Google Scholar, ScienceDirect, Scopus, PubMed, Scientific Information Database and Magiran, using keywords such as hospital, prospective study and foresight, hospital challenges, information technology, economy, human resources, and transformation plan health system. Interviews were conducted with experts in the second phase as a qualitative study using purposive and snowball sampling methods. The most active and well-known experts in the field of health were first identified, selected, and later identified by other experts. The main criteria for selecting them included relevant experience, expertise and ability to conduct research. Sampling continued until data saturation was reached.

\section{Data collection tools}

The literature review was performed using library resources and databases. Semi-structured interviews were conducted with experts to collect data in the qualitative study. The interview questions focused on the factors influencing the hospital's future. Based on the responses, followup questions were asked.

\section{Data collection methods}

This study conducted in-depth, semi-structured, and cross-sectional interviews with 40 hospital specialists. The interviews lasted 30-45 minutes, and every participant was interviewed once. The researcher arranged with the participants the date and place of the interviews. The research setting consisted of medical centers and hospitals affiliated to Iran University of Medical Sciences. The interviews were immediately transcribed after being recorded with the permission of the participants

\section{Data analysis}

The conducted interviews were repeatedly subdivided 
into semantic units and then into smaller meaning units. The codes were then re-read several times to replace the semantic units and main categories by the framework of society, technology, economy, environment, politics, and regulations based on semantic similarity. Efforts were made to avoid interfering with the hypotheses during data analysis. The coding process of the interviews was entirely performed in MAXQDA.

\section{Data reliability of interviews with experts}

The criteria used to determine the reliability of the study data included reliability and stability.

Reliability: Spending time collecting and analyzing the data, selecting experts with different types of expertise and experiences, presenting to public and private hospitals and the Iranian Ministry of Health and Medical Education, meeting the academic community, and selecting participants from different groups enriched the data and findings. In addition, different integration methods used in this study included spatial and human data integration. The present study data were obtained from various sources. Three levels of experts were interviewed and different research methodologies used included content analysis, qualitative study, framework analysis and process analysis.

Stability: Peer review helps validate the data. In this study, three interviews and the results of data analysis were randomly presented to the research team to provide additional comments for the researcher.

Phase 3: Prioritizing driving and effective factors with analytical Hierarchy Process (AHP)

The FAHP was used to determine the effect of individual drivers as per the following steps (16):

Step 1: Designing the pairwise comparison and decision matrices: Identifying appropriate evaluative criteria is the main step in prioritizing the drivers, which was performed by their impact on the future.

Step 2: Defining fuzzy numbers for paired comparison: Table 1 presents the fuzzy numbers used in this study.

The paired matrices designed based on the criteria and hierarchical model were then presented to 15 hospital experts, managers, and officials in Tehran.

3. Calculating the incompatibility coefficients of the matrices completed in Expert Choice.

Step 3: FAHP calculations and method validation:

3-1: The fuzzy composition value of $\widetilde{s_{l}}$ is calculated with i criteria using equation (1).

$\widetilde{s_{l}}=\sum_{j=1}^{m} M_{g i}^{j} \otimes\left[\sum_{i=1}^{n} \sum_{j=1}^{m} M_{g i}^{j}\right]^{-1}, i=1,2,3, \ldots, n$ (1),

in which $\otimes$ denotes the wide multiplication of two fuzzy numbers and each fuzzy number obtained represents the weight of a criterion (or option) relative to another criterion.

3-2. Let $\widetilde{M_{1}}, \widetilde{M_{2}}$ be two triangular fuzzy numbers. The greatness degree of $\widetilde{M_{2}}=\left(l_{2}, m_{2}, u_{2}\right) \geq\left(l_{1}, m_{1}, u_{1}\right)$ is defined using equation (2).

$$
\mu(d)=\left\{\begin{array}{c}
1 \quad m_{2} \geq m_{1} \\
\\
\frac{u_{1}-l_{2}}{\left(u_{1}-m_{1}\right)-\left(m_{2}-l_{2}\right)} l_{1} \geq u_{2}
\end{array}\right. \text { otherwise }
$$

where $\quad \mu(d)=v\left(\widetilde{M_{2}} \geq \widetilde{M_{1}}\right), \widetilde{M_{1}}=\left(l_{1}, m_{1}, u_{1}\right), \widetilde{M_{2}}=$ $\left(l_{2}, m_{2}, u_{2}\right)$.

3-3: The possibility degree of a convex fuzzy number is greater than the possibility degree $\mathrm{k}$ of convex fuzzy number $\widetilde{M}_{l}(i=1,2, \ldots, k)$.

$V\left(\widetilde{M} \geq \widetilde{M_{1}}, \widetilde{M_{2}}, \ldots, \widetilde{M_{k}}\right)=V\left(\widetilde{M} \geq \widetilde{M_{1}}\right)$ and $V(\widetilde{M} \geq$ $\left.\widetilde{M_{2}}\right)$ and .... and $V\left(\widetilde{M} \geq \widetilde{M_{k}}\right)=\min V\left(\widetilde{M} \geq \widetilde{M_{k}}\right), i=$ $1,2, \ldots, k$

3-4: After normalizing $W$, the normalized weight vector is calculated according to equation (4) in which W is a nonfuzzy number.

$$
W=\left(d\left(A_{1}\right), d\left(A_{2}\right), \ldots . d\left(A_{n}\right)\right)^{T}
$$

Compatibility of paired comparison matrices in the AHP is an important issue that should always be considered in the decision process. If the incompatibility coefficient is below 0.1 , judgments will be acceptable. In this study, after performing defuzzification using the center of area method, a conventional hierarchical analysis was conducted to check the compatibility of judgments and FAHP calculations were then performed in Excel.

\section{Ethical considerations}

Ethical issues were observed by receiving a code of ethics (IR.IUMS.REC 2017.9223652202) from the research assistant of Iran University of Medical Sciences. In addition, the participants were briefed on the study objectives and interviewed with the consent of the researcher. The confidentiality of the recorded information was also ensured, and the participants were notified that they could withdraw from the study at their own discretion, although none actually did so.

\section{Results}

Out of the 40 study participants, 28 were male and 12 females. They had an age of 25-65 years and work experience of 1-30 years. Table 2 presents the details of the participants.

Table 1. Fuzzy numbers used in this study

\begin{tabular}{lcccccc}
\hline Fuzzy number & $\tilde{9}$ & $\tilde{7}$ & $\tilde{5}$ & $\tilde{3}$ & $\tilde{1}$ & 1 \\
\hline Definition & Absolute & Very strong & Strong & Low & Equal & Exactly equal im- \\
& importance & importance & importance & importance & importance & portance \\
Triangular fuzzy scale & $(7,9,9)$ & $(5,7,9)$ & $(3,5,7)$ & $(13,5)$ & $(1,1,3)$ & $(1,1,1)$ \\
\hline
\end{tabular}


The content analysis of previous studies and interviews identified six major political, economic, technological, social, cultural and environmental drivers for the future of hospitals (Fig. 2).

\section{Prioritizing the drivers with FAHP}

According to Diagram 1, thirty proponents identified in the previous step were weighted using the FAHP by their impact on the future of hospitals. Incompatibility coefficients of below 0.1 measured for all the paired matrices in Expert Choice confirmed the matrix compatibility. a cloud of economic trends included in policy studies of the health system, as they apply to hospitals with the university and the Ministry of Health as mediators. Haghdoost et al. discussed factors such as technology, the changing age of the pyramid, increasing health literacy and community expectations, environmental challenges and changes, lifestyle changes, community management structures, and the political model of governance in this sector. They found economic changes, value changes, a decline in social capital, changes in family foundations and epidemiological changes to affect the future health of Iranians.

\section{Discussion}

This study considered debates on the economic sanctions

Table 2. The details of the participants

\begin{tabular}{ccccccccccccc}
\hline \multicolumn{2}{c}{ Gender } & \multicolumn{3}{c}{ Age (year) } & \multicolumn{3}{c}{ Education level } & \multicolumn{2}{c}{ Position } \\
\hline Male & Female & $20-30$ & $30-50$ & $>50$ & BA & MA & $\begin{array}{c}\text { PHD \& } \\
\text { MD }\end{array}$ & $\begin{array}{c}\text { Chief executive } \\
\text { officer }\end{array}$ & $\begin{array}{c}\text { Faculty } \\
\text { member }\end{array}$ & $\begin{array}{c}\text { Middle } \\
\text { manager }\end{array}$ & $\begin{array}{c}\text { Expert } \\
\text { Physician }\end{array}$ \\
\hline 28 & 12 & 2 & 28 & 10 & 5 & 10 & 25 & 8 & 10 & 10 & 7 & 5 \\
\hline
\end{tabular}

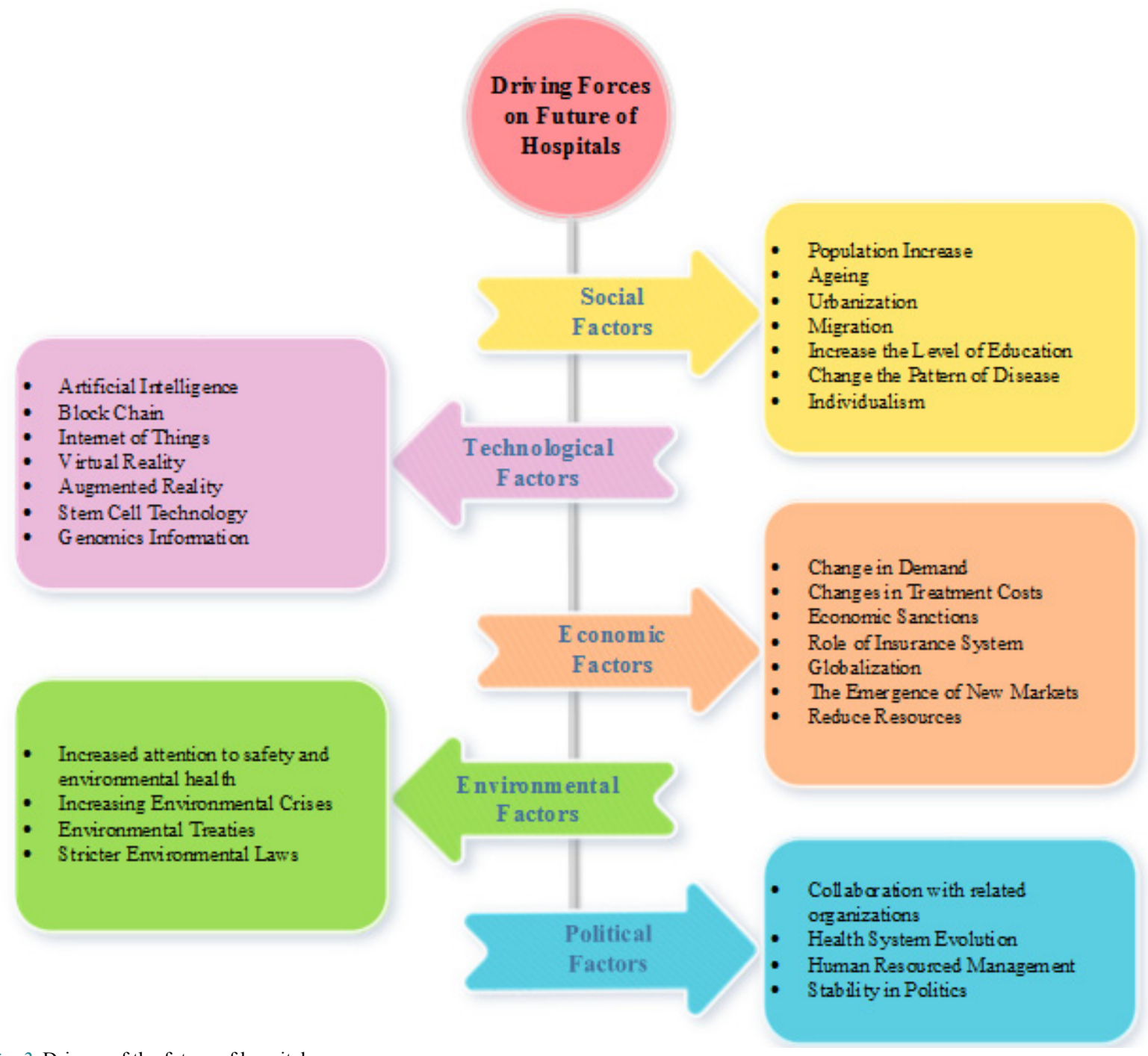

Fig. 2. Drivers of the future of hospitals 


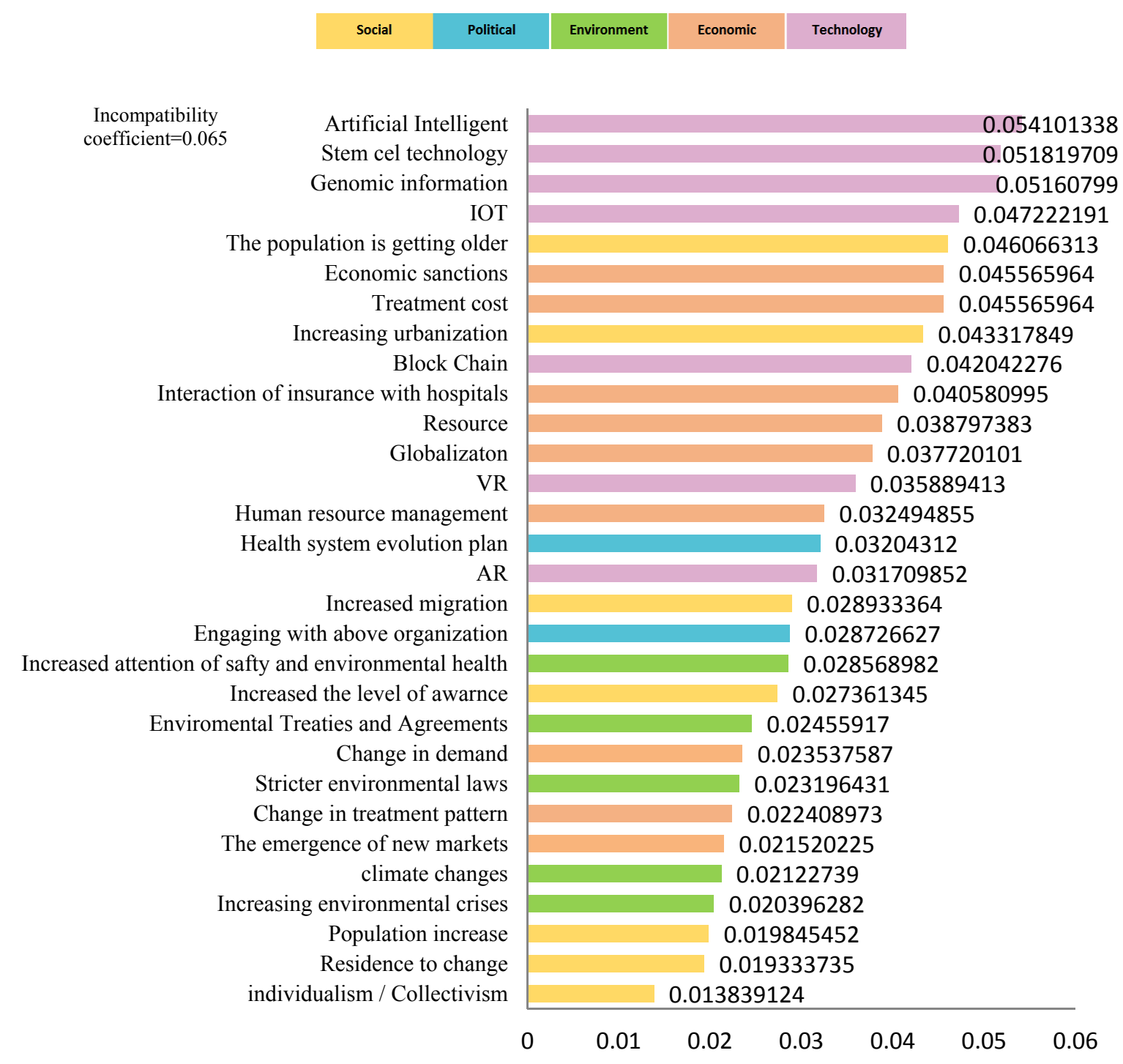

Diagram 1. The degree of the final impact of sub-drivers

This general research in the field of health included hospitals and other health and public health centers (17). This report is consistent with the present study in terms of superlatives such as technology, economy, politics, and the environment, although the right-wing research is included in the policy cloud under the economic sanctions process, whereas the present research considered the economic trend cloud. This discrepancy in results can be explained by the fact that the present research is at a lower level than the research-friendly environment and the economic consequences of sanctions for hospitals are more evident than their political consequences.

Sang reported technology as the main megatrend of the future of Koreans' health, which is consistent with the present results. They emphasized the impact and role of technology on the future of hospitals in the interviews, which was confirmed by the FAHP weighting results. A health report in 2017 by Finch found megatrends such as globalization, sustainability, economy, politics, environment, and technological changes to affect the future of health (13), which is consistent with the present study in terms of the megatrends of technology, economy and politics. Finch identified the environment as a driver of all its dimensions, whereas the present study identified the environment only as an environmental driver of the future according to the experts.

In 2016, Deloitte introduced the demographic megatrends of politics, incoherence, globalization, the digital world and the globalization of technology and industries into the future of health. Drivers such as globalization, technology and politics have also been identified as factors influencing the future of hospitals (18). In 2015, Dadgarpanah identified the factors affecting the future of research on hospital information systems that affected the performance of Milad Hospital's staff in Tehran using a descriptive comparative and causal method and non-random sampling. They prioritized the component of quick decision making which exerted the highest impact on other indicators (19).

A forecast by Ayandehban for Iran 2018 involved seven 
areas, i.e. economy, politics, society, culture, environment, science and technology as well as health. Moreover, 12 different health issues were identified from two groups of experts in all fields and health experts. Health system challenges, mental health issues, health problems due to various pollutions, health problems due to new lifestyle, substance abuse, changing patterns and outbreaks of chronic diseases, health transformation plan, medication issues, health challenges and patients moving towards an aging population, changing people 's preferences in the field of health and expanding sexual risk taking were discussed from the perspective of health experts (20).

Kohsari examined the future of the Iranian health system. They found demographic trends and related phenomena, environment, economy and its impacts on health and injustice, technology and the increasing effects of expectations and costs, national sovereignty and values and beliefs to signficantly affect behavior analyses. Risk factors or health literacy was also found to affect the Iranian health system (21). The environmental, economic and technological trends discussed are consistent with the present findings.

Rahmanian et al. identified six main themes and 13 subthemes as the drivers of the future of hospitals in Iran (22). Leadership included the subthemes of stability, collaborative leadership and incentive leadership; management included management skills, i.e. cognitive and human skills, and adherence to policies; policy training included personnel and managers training, in-hospital policy, out-of-hospital policy and investment in education; creativity and innovation included creativity and scientific innovation and creativity and technological innovation and competition included pptimal subthemes and real competition as the main theme and effective themes in future research(22). Rahmanian et al. examined hospital internal processes and effective management of hospitals in the future, whereas the present study examined outside-oforganization hyperlinks to the future of hospitals. Information technology and politics were presented as two subthemes in this research.

Amin et al. investigated the future of hospital wards (23) and the necessity of expanding clinical and outpatient departments in the fields of chronic diseases and cancers, aging medicine according to population aging processes, rehabilitation after long-term care, sleep clinics, health promotion services such as weight, risk factor management, lifestyle correction and. This research investigated the future of hospitals from a different perspective than that of the present study, i.e. a holistic view.

Aghamohammadi et al. forecast the distribution of hospital beds in Iran between 2006 and 2035 (24). They reported a growing Iranain older adult population until 1414 and found the share of the population aged over 60 of the national population to increase from $7.3 \%$ in 2006 to $17.6 \%$ in 2035 . They also found endocrine, nutritional and metabolic diseases to exert the most signficant effect on mortality in the entire population from 2006 to 2035 with an increase of 1878.52 per thousand. With the status quo being preserved, the number of hospital beds was found to be 160,687 at the highest population fertility and 15,7208 at the lowest. Agha Mohammadi et al. investigated a case on the floor of a hospital, in contrast to the present study, which provided a general and external organizational perspective (24).

\section{Conclusion}

This study identified the factors affecting the future of hospitals based on the results of a literature review and interviews with experts and determined the effect of individual factors using the FAHP. The trends in technological, economic, social and demographic, political and environmental issues were also investigated. A large body of literature has examined the factors affecting the future of the health system as a larger and more general community whose issues are naturally more general and comprehensive; for instance, political debates directly affect the health system, whereas global and national political issues may differently affect hospitals.

\section{Study limitations}

The effective factors obtained under the terms and conditions of the participating experts may be differently reported in other studies with different conditions.

\section{Acknowledgement}

As part of a $\mathrm{PhD}$ dissertation, this study complies with ethical considerations and was approved by Iran University of Medical Sciences, International Campus (IUMS/SHMIs_I/2017/9223652202).

\section{Ethical Issues}

According to ethical considerations, the code of ethics IR.IUMS.REC 2017.9223652202 was obtained from the research deputy of Iran University of Medical Sciences.

\section{Conflict of Interests}

The authors declare that they have no competing interests.

\section{References}

1.Zajac JD. The public hospital of the future. Med J Aust. 2003;179(5):250-2.

2. Hensher M, Fulop N, Coast J, Jefferys E. Better out than in? Alternatives to acute hospital care. BMJ. 1999;319(7217):1127-30.

3. Schofield DJ, Earnest A. Demographic change and the future demand for public hospital care in Australia, 2005 \& nbsp; to 2050. Aust Health Rev. 2006;30(4):507-15.

4. Pickering BW, Litell JM, Herasevich V, Gajic O. Clinical review: the hospital of the future-building intelligent environments to facilitate safe and effective acute care delivery. Crit Care. 2012;16(2):220.

5. Barber SL, Borowitz M, Bekedam H, Ma J. The hospital of the future in China: China's reform of public hospitals and trends from industrialized countries. Health Policy Plan. 2014;29(3):367-78.

6. Goharinezhad S, Maleki M, Baradaran HR, Ravaghi H. A qualitative study of the current situation of elderly care in Iran: what can we do for the future? Glob Health Act. 2016;9:32156.

7. Costa Font J, Sato A. Health systems futures: The challenges of technology, prevention and insurance. Futures. 2012;44(7):696-703.

8. Esmaeili R, Hadian M, Rashidian A, Shariati M, Ghaderi H. Family medicine in Iran: facing the health system challenges. Glob J Health Sci. 2015;7(3):260.

9. Karimi I, Nasiripour A, Maleki M, Mokhtare H. Assessing financing methods and payment system for health service providers in selected countries: designing a model for Iran. J Health Aminist. 2006;8(22):1524. 
10. Aquino RP, Barile S, Grasso A, Saviano M. Envisioning smart and sustainable healthcare: 3D Printing technologies for personalized medication. Futures. 2018;103:35-50.

11. Veenman SA. Futures studies and uncertainty in public policy: A case study on the ageing population in the Netherlands. Futures. 2013;53:4252.

12. Bridle H, Vrieling A, Cardillo M, Araya Y, Hinojosa L. Preparing for an interdisciplinary future: A perspective from early-career researchers. Futures. 2013;53:22-32.

13. Garcia JR, Geher G, Crosier B, Saad G, Gambacorta D, Johnsen L, et al. The interdisciplinarity of evolutionary approaches to human behavior: A key to survival in the Ivory Archipelago. Futures. 2011;43(8):749-61.

14. Goharinezhad S, Maleki M, Baradaran HR, Ravaghi H. Futures of elderly care in Iran: A protocol with scenario approach. Med J Islam Repub Iran. 2016;30:416.

15. Schulz KA, Gstrein OJ, Zwitter AJ. Exploring the governance and implementation of sustainable development initiatives through blockchain technology. Futures. 2020;122:102611.

16. Ravangard R, Bahadori M, Raadabadi M, Teymourzadeh E, Alimomohammadzadeh K, Mehrabian F. A Model for the Development of Hospital Beds Using Fuzzy Analytical Hierarchy Process (Fuzzy AHP). Iran J Public Health. 2017;46(11):1555-62.

17. Mehrolhassani MH, Haghdoost A, Dehnavieh R, Pourhosseini SS . Comprehensive conceptual framework for futures studies in health. Med J Islam Repub Iran. 2019;33:131.

18. van der Laan L, Inayatullah S. Health care foresight: identifying megatrends, convergence and scenarios of ways in which the future can be imagined. Deloitte; 2017.

19. Dadgarpanah Z. Future Studies to Identify Factors Affecting the Hospital Information Systems Staff Performance in Tehran's Milad Hospital. J Manag Futures Res. 2016;27(106):-

20. Molaei M, Talebian H. Futures Studies of Iran Tehran: Research Center for Culture, Arts and Communication; 2017. (In Persian).

21. Kohsari A. Foresight of the Iran Health System [dissertation]. Tehran, Iran: Iran University of Medical Science; 2016. (In Persian).

22. Rahmanian E, Nekoei-Moghadam M, Mardani M. Factors affecting futures Studies in hospitals: A Qualitative Study. J Qual Res Health Sci. 2019;7(4):361-71.

23. Amin E, Ghasemi E, Haghdoost AA, Hashemian F, Sabermahani A. The Hospital Wards of the Future Framework. J Rafsanjan Uni Med Sci Health Serv. 2016;15(7):607-20.

24. Aghamohamadi S, Jahangiri K, Hajinabi K, Masoudi Asl I, Dehnavieh R. Foresight for Inpatient Beds in Hospitals of Iran: Vision 2035. J Health Administ. 2018;21(71):23-36. 